\title{
Ein echtes Public Health-Dokument
}

\section{Jean Martin}

Dr. med., Redaktionsmitglied

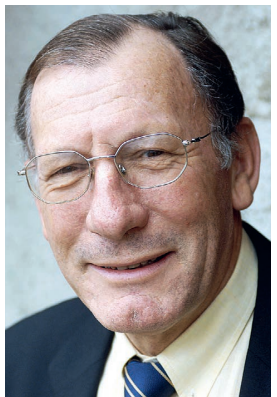

«Immer und überall war der Zugang zu einer besseren Pflege aus wirtschaftlichen, geographischen, kulturellen und ideologischen Gründen ungleich und ungerecht. Die Gesundheitsbedürfnisse von Einzelpersonen und Bevölkerungsgruppen waren schon immer und überall ungleich und ungerecht. Dafür zeichnen genetische, soziale und umweltspezifische Ursachen verantwortlich ...»

Starke Worte - sie beschreiben jedoch einen andauernden Zustand, der als primum movens für Public Health steht, also für die Gesamtheit der Handlungen, die auf eine bessere Gesundheit der Bevölkerung abzielen. Ganz ohne abwerten zu wollen, wäre daran zu erinnern, dass sich der Beitrag, den die Gesundheitssysteme leisten, zwischen 15 und 20\% bewegt. Der grösste Teil jener Faktoren, die einen positiven oder einen negativen Einfluss auf unsere Gesundheit zeitigen, ist anderen, öffentlichen wie privaten Lebensbereichen zuzuordnen. Das vorstehende Zitat ist dem Bericht zur Gesundheitspolitik - 2018-2022 entnommen, den der Waadtländer Staatsrat soeben verabschiedet hat [1, 2]. Eine gute Lektüre für all jene, die mit den Herausforderungen befasst sind, denen sich unsere Gesundheitsfürsorge inskünftig, vor allem in Bezug auf die Nachhaltigkeit und die Finanzierung des Systems, das Miteinander und die Ausbremsung einer Tendenz zur Entsolidarisierung, zu stellen hat. Dazu einige Eckpunkte:

Macht und Grenzen der Medizin. "Auf der einen Seite erscheint uns die Medizin heute stärker denn je dank der technologischen Entwicklungen. Gleichzeitig aber bleibt der Einklang zwischen dem Zugang zur Pflege und den Gesundheitsbedürfnissen weiterhin eine grosse Herausforderung. Davon ist auch das Gleichgewicht der Investitionen im Gesundheitswesen im Vergleich zu Investitionen in anderen Bereichen betroffen. Die stabile Entwicklung des Systems ist bedroht durch ein ständiges Zurücksetzen der diagnostischen und therapeutischen Grenzen. Zwar waren diese Grenzen bis jetzt wirtschaftlich und ethisch erträglich. Heute stellt sich die Frage, ob alles, was machbar ist, auch zu wünschen ist.»

Gesunde intersektorielle Politik. In der Einleitung zum Bericht schreibt der Direktor des Gesundheitsdepartements: «Um diese Ziele zu erreichen, gilt es, die Verbindung zu den anderen Strategien des Staatsrats zu knüpfen. Auch sie setzen entschieden auf die Ge- sundheit der Bevölkerung.» Dies erinnert mich an einen vor langer Zeit notierten Satz, der bislang in keinem Land richtig umgesetzt wurde. «We don't need a public health policy, we essentially need a healthy public policy" (wir brauchen keine Gesundheitspolitik, sondern vielmehr eine gesunde staatliche Politik). Das ganze regierungsseitige Vorgehen bedarf einer Gesamtsicht, der Umsetzung «interministerieller» Strategien. Und dies idealerweise in der gesamten Wirtschaft, um die Gesundheit eher zu fördern als ihr zu schaden. Im Bericht spricht Wirkungsfeld 1 von der «Konsolidierung der departementsübergreifenden Zusammenarbeit, um so das Schadstoffrisiko zu mindern" und davon, "den Unternehmen Werkzeuge zu geben, damit sie der Gesundheit am Arbeitsplatz besser begegnen können». An anderer Stelle wird die Notwendigkeit unterstrichen, "Gesundheit als Massnahme im [generellen] Rahmen der Kinder- und Jugendpolitik zu artikulieren".

Autonomie von Patienten/Personen. Der Bericht engagiert sich stark: «Wir alle müssen Subjekt und nicht Objekt der Pflege bleiben, autonom sein und bleiben können, dabei gleichzeitig aber begleitet werden. Diese Autonomie ist ein Grundrecht, das es nicht nur zu schützen, sondern auch zu bevorzugen gilt.» Dies gilt gleichermassen für Kinder und Jugendliche, für eine "verstärkte Rücksichtnahme auf deren Recht auf Selbstbestimmtheit, das Respektieren ihrer Werte».

«Die richtige Pflege.» «Richtige Pflege antwortet auf das Risiko der sozialen Ungleichheit und wendet einen ausgewogenen Universalismus an [Definition auf Seite 14], respektvoll gegenüber der aufgeklärten Wahl der Person, die in deren Lebensumfeld so weit als möglich Anwendung findet. Sie ist von keinerlei sozialer, geschlechts- oder kulturspezifischer Konditionierung abhängig. Richtige Pflege ist zuallererst eine Beziehung und erst in zweiter Linie eine Dienstleistung." Qualität der Pflege. Der hier "verfügbare Platz» lässt ein näheres Eingehen auf Wirkungsfeld 7 nicht zu. Letzteres geht jedoch auf sechs Hauptbereiche ein: Sicherheit, Reaktivität, Wirksamkeit, Effizienz, Gerechtigkeit und «Patientenzentriertheit» - bzw. Angemessenheit. Daran knüpft sich eine pädagogische Absicht: «die Entwicklung von ethischen, klinischen, sozialen und verantwortungsspezifischen Kompetenzen bei den Fachkräften». 